\title{
Spectroscopy and Light Emitting Diodes Based System in Characterizing External Beam Therapy 3 Films for Solar Ultraviolet Measurement
}

\author{
Wan Insaniah Saleha AHMAD SHAH and Ahmad Fairuz OMAR* \\ School of Physics, Universiti Sains Malaysia, 11800 USM, Penang, Malaysia \\ *Corresponding author: Ahmad Fairuz OMAR_E-mail: fairuz_omar@usm.my
}

\begin{abstract}
Gafchromic external beam therapy 3 (EBT3) film has widely been used in medical field applications. Principally, the EBT3 film's color gradually changes from light green to darker color under incremental exposures by ionizing or even non-ionizing ultraviolet (UV) radiation. Peak absorbance of the EBT3 film can be used to predict absorbed doses by the film. However, until today, related researches still rely on spectrometers for color analysis of EBT3 films. Hence, this paper presents a comparative analysis between results produced by the spectrometer and a much simpler light-emitting diode-photodiode based system in profiling the color changes of EBT3 films after exposure by solar UV radiation. This work has been conducted on a set of 50 EBT3 samples with incremental solar UV exposure (doses). The wavelength in the red region has the best sensitivity in profiling the color changes of EBT3 films for low solar UV exposure measurement. This study foresees the ability of blue wavelength to profile films with a large range of solar UV exposure. The LED (light emitting diode)-based optical system has produced comparable measurement accuracies to the spectrometer and thus, with a potential for replacing the need for a multipurpose spectroscopy system for simple measurement of light attenuation.
\end{abstract}

Keywords: External beam therapy 3; light emitting diode; solar ultraviolet; visible absorbance spectroscopy

Citation: Wan Insaniah Saleha AHMAD SHAH and Ahmad Fairuz OMAR, "Spectroscopy and Light Emitting Diodes Based System in Characterizing External Beam Therapy 3 Films for Solar Ultraviolet Measurement," Photonic Sensors, 2020, 10(1): 3444.

\section{Introduction}

The radiochromic film is an important tool in the medical field application, which is commonly used to verify dose distributions in highly conformal radiation therapy such as intensity-modulated radiation therapy (IMRT), volumetric modulated arc therapy (VMAT), and X-ray [1-3]. Since the last decade, more researches have been conducted on the applicability of various types of radiochromic films in measuring ultraviolet (UV) exposure [4-6]. One of them is the gafchromic external beam therapy
3 (EBT3) film. The blue colored polymer is formed when the active component in the film is exposed to radiation [7]. The color changes of EBT3 films have been proven to be highly correlated with the absorbed UV exposure by the films [8-17]. For the color analysis of the film through the visible absorbance spectroscopy technique, researchers found that the wavelength in the red region of the spectrum has the highest absorption [17-19]. The absorption spectrum for the EBT3 film has two distinctive peaks, at $633 \mathrm{~nm}$ and $582 \mathrm{~nm}$. However, the absorption peak at $633 \mathrm{~nm}$ provides a higher sensitivity than the peak at

Received:19 November 2018 / Revised: 17 May 2019

(C) The Author(s) 2019. This article is published with open access at Springerlink.com

DOI: $10.1007 / \mathrm{s} 13320-019-0565-6$

Article type: Regular 
$582 \mathrm{~nm}$ [17]. Choosing a light source with a wavelength at the maximum absorption will provide the highest sensitivity for the EBT3 film measurement [20]. In ionizing radiation application, the wavelength at $635 \mathrm{~nm}$ has been employed to accurately measure the absorption of the film for doses level less than $10 \mathrm{~Gy}$. However, for doses level larger than $10 \mathrm{~Gy}$, the absorption saturates due to a small amount of light transmitting through the film and limits the sensitivity of the spectrometer [21]. For the same reason, UV exposure measurement using the EBT3 film can be made up to $30000 \mathrm{~mJ} / \mathrm{cm}^{2}$ [9]. Besides spectroscopy based color measurement, the flatbed scanner is another important optical tool commonly used to profile the color changes of the EBT3 film. The red channel produces the highest sensitivity in transmission and reflection modes of the scanner while the blue channel shows the lowest sensitivity and cannot be used because of the effect of yellow dye [19]. In an experiment, the optical density (OD) of the EBT3 film was measured by two scanners, and the larger values for the red channel was gained which showsed more sensitivity, hence, better dose measurement results were compared with that in the RGB spectrum analysis [18]. The UV-visible absorption spectrum, however, has been identified to be more sensitive to changes in dose (i.e., in relation to a polymer content of the EBT3 film) compared with the flatbed scanning method [21].

The application of the scanner is time consuming due to the required orientation and post-exposure analysis of the film. For example, the transmission mode scanning requires continuous scanning orientation and less exposure to room light to reduce uncertainty in measured dose. Meanwhile, the reflection scanning mode shows a good stability to room light, but film orientation has a large uncertainty in measured dose [22]. Additionally, reflective scanning analysis is an economic method for the EBT3 film compared with the transmission method. In the reflection scanning mode, there is no difference between the landscape and portrait orientation. Likewise, the single scan approach is recommended, although single and multiple scans do not show any significant difference as long as the size and number of samples still fit in the scanning area [18]. In contrast, the result differs for peeled-off EBT3 film (i.e., one polyester cover above the active layer is removed). The responses are different for different scanning directions (i.e., landscape and portrait scan) [2]. Furthermore, the orientation dependence with respect to the front and back sides of the film is completely eliminated in the EBT3 film due to the symmetrical layer of the film [1].

Until today, the flatbed scanner [9, 10, 18, 23-25] and visible spectroscopy $[7,8,17,21]$ are considered to be the most acceptable optical tools in analyzing the color changes of the radiochromic film and red channel/wavelength to be the most sensitive in the application. Hence, to utilize only a single red wavelength from a spectrometer or a single red channel from a scanner is not a cost-effective measure. Furthermore, this instrumentation requires post-processing in the film analysis. Therefore, this paper presents an innovative application of several colored light emitting diodes (LEDs) as an illumination light source with a single photodiode as the detector. The single-colored LED as a light source can replace the tungsten halogen lamp, which is commonly used in the broad spectral spectroscopy analysis. In addition, the LED emits a narrow spectrum of light and can operate at the low temperature and voltage. The purpose of this study is to offer comparative analysis between the visible absorbance spectroscopy and LED-based optical system in profiling the color changes of EBT3 films in the measurement of natural solar UV exposure. The result of this research may assist in the development of the miniaturizing and portable personal UV dosimeter, partly, as a promising tool to monitor the possible harmful solar UV overexposure on humans. The LED-photodiode system offers a low-cost and rapid alternative in the characterization 
and the measurement of EBT3 films' colors in relation to the solar UV exposure.

\section{Materials and methods}

A piece of EBT3 film $(20.32 \mathrm{~cm} \times 25.4 \mathrm{~cm}$ dimension) was cut into 50 small pieces with a $1 \mathrm{~cm} \times 2 \mathrm{~cm}$ dimension. All the films were placed simultaneously on a white mahjong paper to ensure uniformity in the potential back reflected light [26]. The experiment was conducted during a clear sky between 12:30 pm and 1:45 pm (1 hour and 15 minutes) at the rooftop of School of Physics, Universiti Sains Malaysia. The selected time, duration of experiment, and location were based on the intention to obtain the maximum UV exposure towards the surface of the films. The weather was hot with temperature of $30{ }^{\circ} \mathrm{C}-32{ }^{\circ} \mathrm{C}$ during the experiment with the irregular and brief appearance of clouds. Solarmeters (models 4.0 UVA and 6.0 UVB) were used in the experiment to record the total UV irradiance (UVA + UVB). UVA meter had an irradiance measurement range between 0 and $199.9 \mathrm{~mW} / \mathrm{cm}^{2}$ with a resolution of $0.1 \mathrm{~mW} / \mathrm{cm}^{2}$ while the UVB meter irradiance range was between 0 and $19.99 \mathrm{~mW} / \mathrm{cm}^{2}$ with a resolution of $0.01 \mathrm{~mW} / \mathrm{cm}^{2}$. The UV meters were placed close to EBT3 films during exposure. During the solar UV measurement, the irradiance was measured every 30 seconds until the 90th second and averaged. For each 90 seconds, a film was collected from the mahjong paper and placed in a black envelop to keep the film away from the further exposure by UV irradiance. This step was repeated for the next film until all 50 films were completed. The total exposure on each film was based on the accumulation of averaged UV irradiance over a period of 90 seconds. Each film represented an incremental value of UV exposure with the maximum recorded value of $32801.7 \mathrm{~mJ} / \mathrm{cm}^{2}$. However, since the UV measurement was made only in the interval of 30 seconds, any drastic changes in irradiance might not be captured. Hence, it was important to take note of the sky condition and the irradiance gradient throughout the experiment to avoid any discrepancies in total UV exposure in relation to the EBT3 color.

The color of the films was measured by using two optical systems. The first was through the visible absorbance spectrometer and the second was through a simplified system with a LED as a light source and a photodiode as the detector. Visible absorbance spectra of the films were measured by using Ocean Optics QE65000 spectrometer and tungsten halogen lamp (HL-2000) as the illumination light source. In spectroscopy measurement, darker films, due to the higher UV exposure, result in the higher absorbance. For the LED-based system, seven low power $5 \mathrm{~mm}$ LEDs with different emission colors were used as the light sources. The LEDs were red (HLMP-EG08-WZ000) with a peak emission $\left(\lambda_{\text {peak }}\right)$ at $635 \mathrm{~nm}$, red-orange (HLMP-EH15-TW000) with $\lambda_{\text {peak }}$ at $616 \mathrm{~nm}$, orange (HLMP-EL15-SV000) with $\lambda_{\text {peak }}$ at $596 \mathrm{~nm}$, yellow (HLMP-C515) with $\lambda_{\text {peak }}$ at $563 \mathrm{~nm}$, green (LC503FPG1-15Q-A3-00011) with $\lambda_{\text {peak }}$ at $519 \mathrm{~nm}$, cyan (HLMP-CE16-WZQDD) with $\lambda_{\text {peak }}$ at $501 \mathrm{~nm}$ and blue (HLMP-CB2B-VW0DD) with $\lambda_{\text {peak }}$ at $462 \mathrm{~nm}$ as presented in Table 1 .

Table 1 Properties of red, green, blue, orange, red orange, cyan, and yellow LEDs used in this experiment.

\begin{tabular}{ccccc}
\hline Model & Wavelength (nm) & $\begin{array}{c}\text { Maximum } \\
\text { intensity }(\mathrm{mcd})\end{array}$ & $\begin{array}{c}\text { Maximum } \\
\text { current }(\mathrm{mA})\end{array}$ & $\begin{array}{c}\text { Typical } \\
\text { voltage }(\mathrm{V})\end{array}$ \\
\hline Red & 635 & 16000 & 50 & 1.9 \\
Green & 535 & 34000 & 25 & 3.2 \\
Blue & 464 & 7200 & 30 & 3.2 \\
Orange & 592 & 6300 & 50 & 2.02 \\
Red orange & 621 & 7200 & 50 & 1.94 \\
Cyan & 502 & 16000 & 30 & 3.2 \\
Yellow & 568 & 490 & 30 & 2.2 \\
\hline
\end{tabular}

The attenuation of LED emission through the film was measured by using TSL257 (TAOS Inc.) which was a light to the voltage optical converter that combined a photodiode and a transimpedance amplifier. TSL257 had a broad spectral sensitivity across and beyond the visible region (i.e., between 
$300 \mathrm{~nm}$ and $1100 \mathrm{~nm}$ ) as shown in Fig. 1. For this experiment, darker films resulted in higher light attenuation through the film which subsequently led to the lower output voltage from the photodiode. Peak sensitivity of an optical system is defined based on the peak emission wavelength of the LED, which is strongly related to the peak responsivity of the detector. The optical measurement achieves the maximum efficiency by matching the detector response to the emission spectra of the light source. Hence, it is important to select the light source and optical sensor (detector) that have similar optical characteristics [27]. Each LED selected for this experiment had a peak emission wavelength that fell within the spectral sensitivity of the detector. Table 2 lists the electrical characteristics of TSL257. From the table, among the tested emission wavelengths, $645 \mathrm{~nm}$ produced a higher sensitivity compared with other wavelengths (i.e., $428 \mathrm{~nm}, 470 \mathrm{~nm}$, and $656 \mathrm{~nm}$ ). Hence, it is important to set the output voltage of the detector to a standard initial voltage prior to the experiment, so that the attenuation degree for all LEDs illumination through the film can be impartially compared.

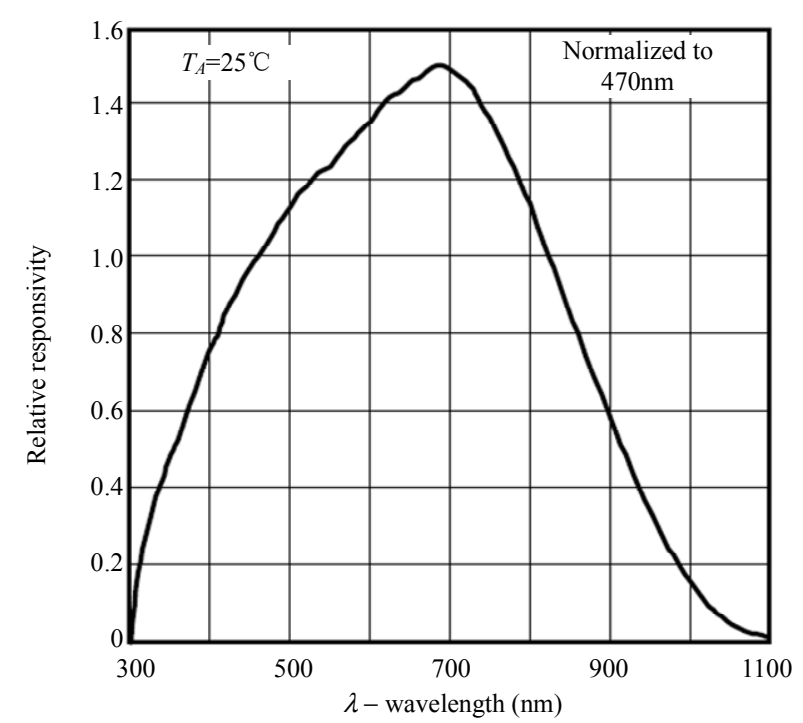

Fig. 1 TSL257 photodiode spectral responsivity [28].

The experimental setup based on the LED-photodiode system is illustrated in Fig. 2 while the schematic diagram of the entire system is shown in Fig. 3. Prior to the experiment, the unexposed EBT3 film was used as a reference sample to calibrate the system by adjusting the intensity of the LED through the film until the output voltage of the detector was set to $4.5 \mathrm{~V}$. This was to ensure that the maximum possible intensity through the film was lower than the detector saturation level (i.e., $5 \mathrm{~V}$ ). After the calibration, each exposed EBT3 film was placed in a film's holder, and the measurement was recorded by using a multimeter in the unit of voltage (V). A similar method was employed on all seven LEDs. In this experiment, a portion of the illuminated light from the LED was absorbed by the film while the rest transmitted through the film. A portion of the transmitted light was directed to the detector through the collimator. Darker films absorbed more light and subsequently attenuated the intensity of the light to the detector.

Table2 Specifications of TSL257 [28].

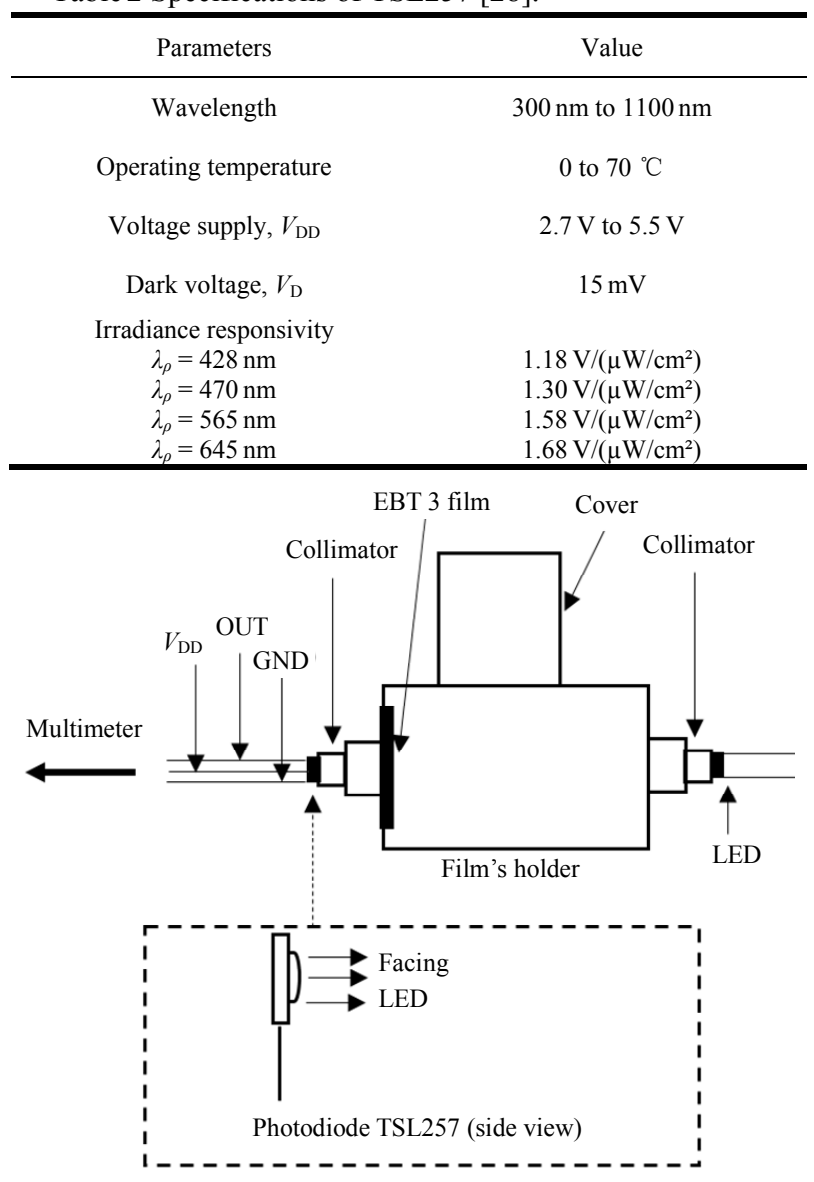

Fig. 2 Side views of LED and TSL257 photodiode which were attached to the collimators. 


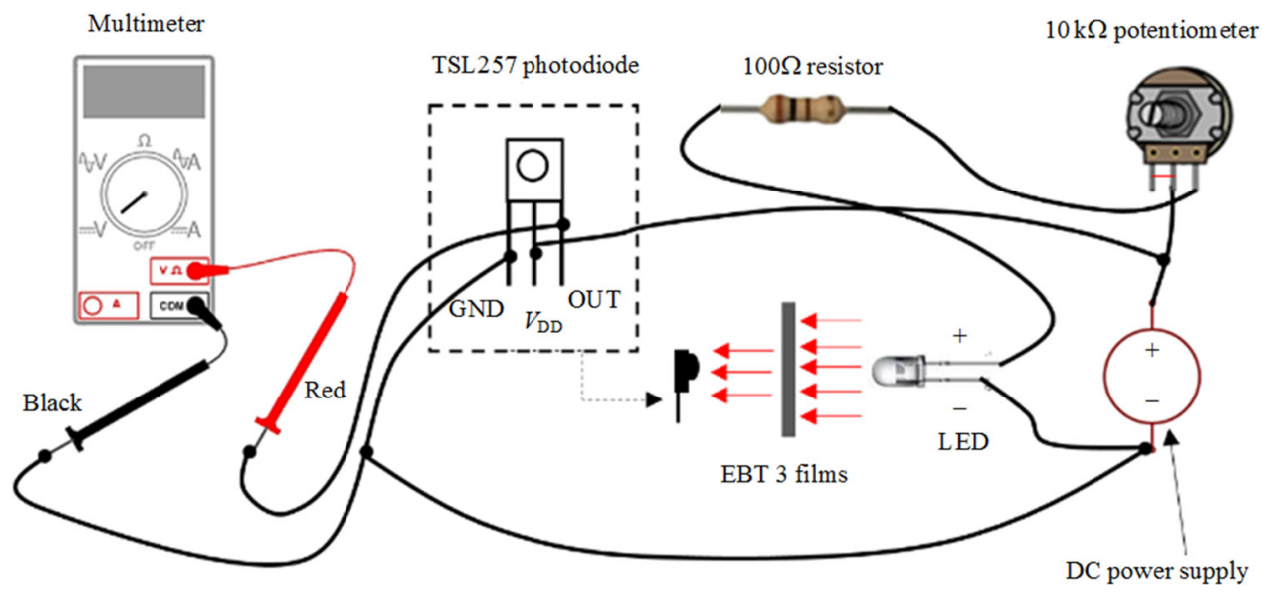

Fig. 3 Schematic diagram of the LED-photodiode based measurement.

\section{Results and discussion}

The increment in the level of the EBT3 films' darknesses is highly proportional to the level of solar UV exposure on the films. Based on the observation, the original color of the film (light green) gradually darkens (dark blue) during the exposure. The change in color indicates that the crystalline diacetylenes monomer, which is lithium salt of pentacosa-10, 12-diyonic acid (LiPCDA) in the active layer of EBT3 film undergo 1,4-polymerization when exposed to UV or ionizing radiation [20]. The crystalline diacetylenes monomer (i.e, colorless long-chained molecules) polymerized and formed linear long chained polymer (i.e, colored polymer). The colored polymer is in blue color. The colored polymer formed can exhibit at least three distinct color phases which are the bluish-green phase, blue phase, and the red phase polymer. The reason for the blue color appearance is due to the dominant blue phase polymer in the active layer of the EBT3 film [7]. These colored polymer chains grow in length with the level of exposure. In other words, the colored polymer chains concentration increases with increasing level of exposure and becomes increasingly dark [21]. The formed polymeric chains are known as polydiacetylene dye polymers (polyPCDA). The blue polyPCDA causes the film to absorb light in the red part of the visible spectrum [7].

Figure 4(a) shows the visible absorption spectra of 50 exposed EBT3 films and general image representation of EBT3 colors under different exposures. Higher solar UV exposure causes the color transformation of EBT3 films from light green to darker blue. Through the absorbance spectroscopy technique, darker films will result in higher absorbance reading. The range of UV exposure recorded in the experiment was between $584 \mathrm{~mJ} / \mathrm{cm}^{2}$ and $32801.7 \mathrm{~mJ} / \mathrm{cm}^{2}$ with zero exposure film used as the reference during absorbance calibration. Based on the literature, selecting a light source centered at $\lambda_{\rho}$ will provide the highest sensitivity for the film analysis. Furthermore, the absorbance level is dependent on the wavelength of illumination light [20]. The absorption in the blue region of visible spectra (approximately below $500 \mathrm{~nm}$ ) is recorded to be the lowest.

According to the filter and color subtraction process, when white light illuminates on the blue filter, the blue wavelength of light will pass through the filter while the rest of the wavelength will be absorbed by the film. This is proven by the color subtraction process concept where white light can be formed by the combination of primary colors, i.e., red, green, and blue. Based on the color subtraction 
process, it can be expressed as

$$
\text { Blue }=\text { Blue filter }=\text { White }-(\text { Red }+ \text { Green })
$$

The EBT3 film is dominated by the optical properties of the blue phase polymer. By applying the concept of the filter and color subtraction process, a large portion of blue light can transmit through the blue colored polymer dye in the EBT3 film while the rest of the wavelength will be absorbed. Therefore, the blue light has the lowest sensitivity or response to the EBT3 film due to the lowest absorption. This explains the lowest absorbance (0 to $0.6 \mathrm{OD}$ ) for wavelengths lower than $490 \mathrm{~nm}$ [Fig. 4(a)]. Meanwhile, the red region of the visible spectra carries the highest responsivity with the maximum absorbance reaching to $2 \mathrm{OD}$, while the green region has the maximum absorbance slightly above 1.8OD. The red light has the highest reaction towards the blue-colored polymer dye EBT3 film compared with green light. This clarifies that the exposure-response curve for the EBT3 film for the absorption peak at $630 \mathrm{~nm}$ (red light) offers a higher sensitivity than the peak at $580 \mathrm{~nm}$ (green light). This can be further observed in Figs. 4(b) and 4(c) which show the relationship between the absorbance and UV exposure for two peak absorbances i.e., $580 \mathrm{~nm}$ and $630 \mathrm{~nm}$, respectively. The findings also show that the absorbance measurement can be made regardless of the film's orientation (i.e., either from the front or from back side).

The relationship between the absorbance and UV exposure can be represented by using a different function in order to obtain the best regression fit along with the data with a coefficient of determination, $R^{2}$, preferably above 0.98 . Since the wavelength within the red region is highly sensitive to EBT3 colors, by obeying Beer-Lambert law of absorption, its responses curve will have an earlier inclination towards an asymptote. Unlike wavelengths within less sensitive regions such as far red and blue, the response to films with high UV exposure is much lower and can even be profiled by using a simple linear function.
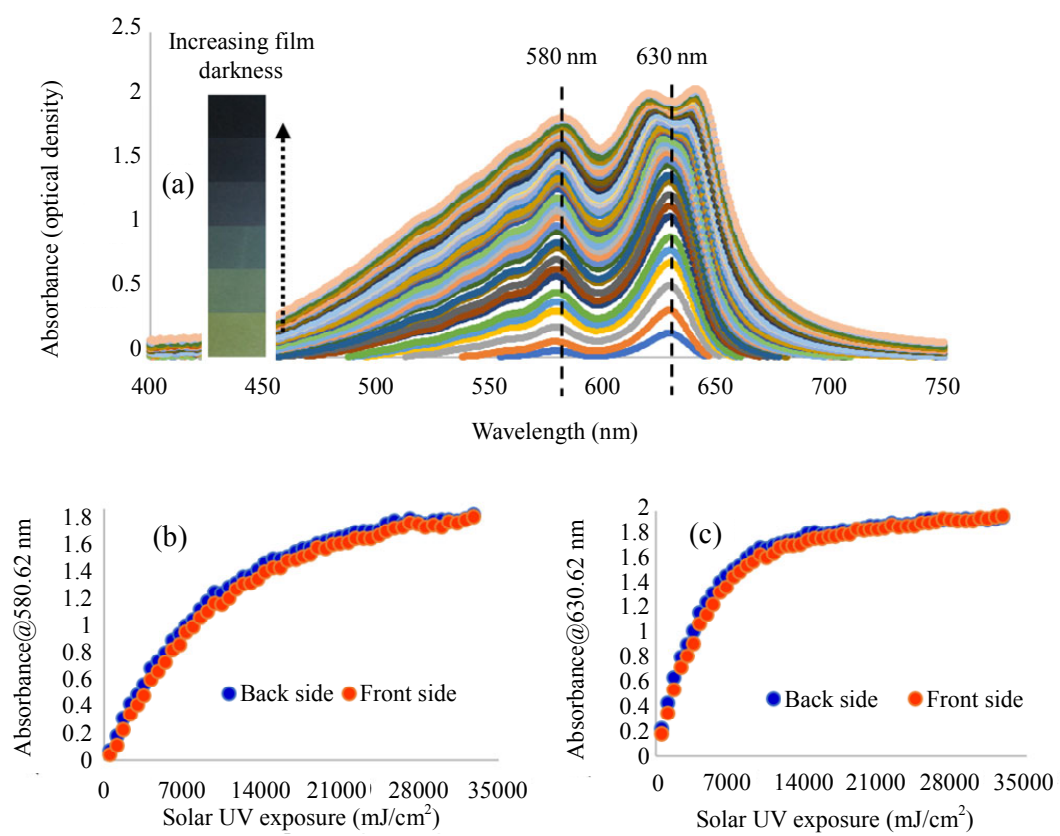

Fig. 4 Spectral response of EBT3 film: (a) visible absorption spectrum of gafchromic EBT3 films irradiated under incremental solar ultraviolet exposure between $584 \mathrm{~mJ} / \mathrm{cm}^{2}$ and $32801.7 \mathrm{~mJ} / \mathrm{cm}^{2}$. The relationship between the solar ultraviolet exposures $\left(\right.$ in $\mathrm{mJ} / \mathrm{cm}^{2}$ ) and films' absorbances measured from both sides of the films at two peak absorbance wavelengths: (b) $580 \mathrm{~nm}$ and (c) $630 \mathrm{~nm}$.

Figure 5 shows the graphical representation of absorbance against solar UV exposure for the selected wavelengths. It can be observed that the best response for lower UV exposure (i.e., less than 
$10000 \mathrm{~mJ} / \mathrm{cm}^{2}$ ) is produced by using films' absorbances at $620 \mathrm{~nm}$ and $630 \mathrm{~nm}$. But the sensitivity of these wavelengths starts to decline at a higher UV exposure since the curve starts to move gradually closer towards asymptote. The sensitivity curve of the nonlinear function starts to decline for wavelengths lower than $620 \mathrm{~nm}$ and higher than $630 \mathrm{~nm}$, until a point where the response starts to linearise. The wavelength $650 \mathrm{~nm}$ displays the best linear response with the high responsivity compared with the wavelength at the farther red region (i.e., $670 \mathrm{~nm}$ and $690 \mathrm{~nm}$ ) and blue region (i.e., $450 \mathrm{~nm}$, $460 \mathrm{~nm}$, and $490 \mathrm{~nm}$ ) with lower regression fit with $R^{2}$ approximately 0.96 .

Table 3 lists all the functions and coefficients of determination, where $R^{2}$ is for the response curve by each wavelength. The function is selected based on its ability to generate good regression with a coefficient of determination preferably above 0.98 .

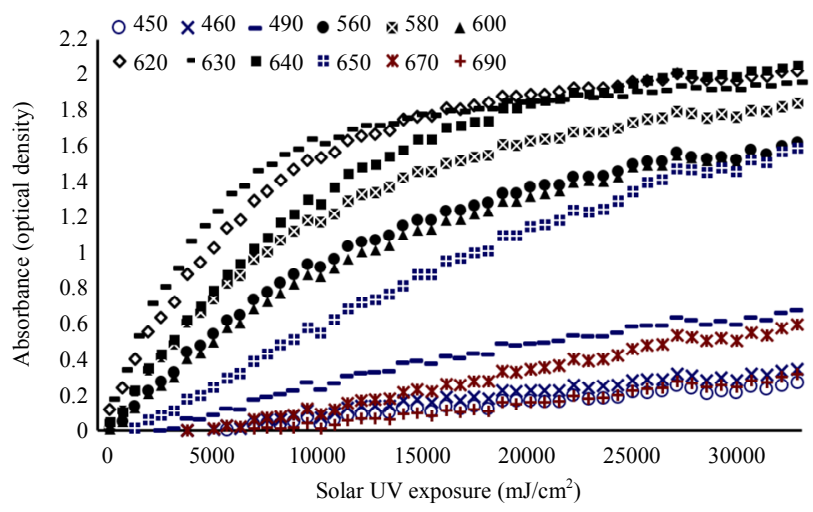

Fig. 5 Response curve obtained from the relationship between the solar ultraviolet exposures (in $\mathrm{mJ} / \mathrm{cm}^{2}$ ) and absorbances of the films developed by using selected wavelengths within visible spectra.

Table 3 Summary of functions and coefficients of determination obtained from the relationship between the solar ultraviolet exposures $\left(\mathrm{mJ} / \mathrm{cm}^{2}\right)$ and absorbances of the films developed by using selected wavelengths within visible spectra.

\begin{tabular}{|c|c|c|c|c|}
\hline Functions & Wavelength (nm) & Relationship & Coefficients & $R^{2}$ \\
\hline \multirow{3}{*}{$y=m x+c$} & 450 & Linear & $m=1 \times 10^{-5}, c=-0.0568$ & 0.965 \\
\hline & 460 & Linear & $m=1 \times 10^{-5}, c=-0.0522$ & 0.965 \\
\hline & 490 & Linear & $m=2 \times 10^{-5}, c=-0.0105$ & 0.961 \\
\hline \multirow{9}{*}{$y=a+\frac{b}{x}+c \ln x$} & 510 & Non-linear & $a-3.63, b=501.2141, c=0.4365$ & 0.990 \\
\hline & 530 & Non-linear & $a=-4.2381, b=555.8915, c=0.5197$ & 0.994 \\
\hline & 550 & Non-linear & $a=-4.7107, b=562.4108, c=0.5934$ & 0.996 \\
\hline & 560 & Non-linear & $a=-4.9821, b=571.2694, c=0.6344$ & 0.997 \\
\hline & 570 & Non-linear & $a=-4.9614, b=522.4779, c=0.6441$ & 0.996 \\
\hline & 580 & Non-linear & $a=-4.9726, b=468.5155, c=0.6593$ & 0.994 \\
\hline & 590 & Non-linear & $a=-5.483, b=664.1099, c=0.692$ & 0.997 \\
\hline & 600 & Non-linear & $a=-5.1912, b=644.2356, c=0.6519$ & 0.996 \\
\hline & 610 & Non-linear & $a=-5.4731, b=611.8412, c=0.706$ & 0.997 \\
\hline \multirow[t]{2}{*}{$y=a+b e^{c x}$} & 620 & Non-linear & $a=2.01482, b=-2.0737, c=-0.000139611$ & 0.999 \\
\hline & 630 & Non-linear & $a=1.90941, b=1.91843, c=-0.000187755$ & 0.996 \\
\hline$y=a+\frac{b}{x}+c \ln x$ & 640 & Non-linear & $a=-5.9382, b=607.6033, c=0.7758$ & 0.993 \\
\hline$y=m x+c$ & 650 & Linear & $m=5 \times 10^{-5}, c=0.0049$ & 0.983 \\
\hline \multirow[t]{2}{*}{$y=m x+c$} & 670 & Linear & $m=2 \times 10^{-5}, c=-0.0948$ & 0.993 \\
\hline & 690 & Linear & $m=1 \times 10^{-5}, c=-0.0828$ & 0.985 \\
\hline
\end{tabular}


Regression fit generated by using absorbance with the peak emission wavelength at $450 \mathrm{~nm}$, $460 \mathrm{~nm}$, and $490 \mathrm{~nm}$ however was best recorded by using a linear function with a slightly lower coefficient of determination around 0.96. This may be caused by the lower sensitivity of the range of wavelengths in profiling the color changes of external beam therapy 3 films. The logarithmic function was used to generate regression fit for the wavelengths between $510 \mathrm{~nm}$ and $610 \mathrm{~nm}$; and wavelength at $640 \mathrm{~nm}$. An exponential function was used for wavelengths of $620 \mathrm{~nm}$ and $630 \mathrm{~nm}$. Among all the linear functions, the wavelength of $650 \mathrm{~nm}$ was able to generate the best responsivity based on its steeper slope $(m)$. The purpose of presenting these functions in relation to solar UV exposure and absorbance is to identify the performance and accuracy of wavelength for the measurement of solar UV exposure.

The films color measurement by using a visible absorbance spectroscopy technique was repeated by using the LEDs-photodiode based system. The purpose of this experiment was to introduce a more cost-effective technique for simple optical transmittance/absorbance measurement that was capable of producing comparable results to a much complex spectroscopy system. Hence, instead of using a broad-spectrum tungsten halogen lamp, this section introduces the application of single colored LEDs. A single photodiode (detector) was used as a replacement of the spectrometer in detecting the transmitted LED light through the EBT3 film. Figure 6 shows the spectra of seven visible LEDs used in this experiment. These are the typical peak emission wavelengths of visible LEDs that are available in the market. The spectra were measured by using QE65000 spectrometer through direct emission. The intensity of the LEDs was adjusted until the intensity displayed by the spectroscopy system was standardized to approximately 40000 a.u.

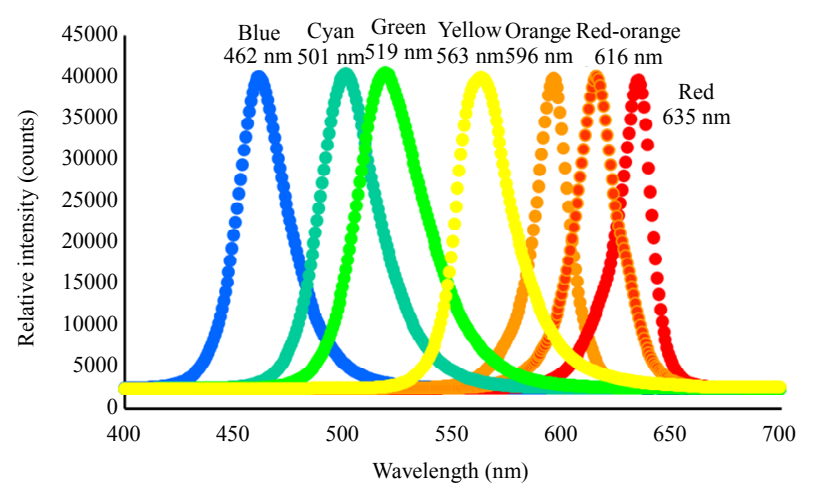

Fig. 6 Visible spectra of seven light emitting diodes with the peak emission wavelengths at $462 \mathrm{~nm}, 501 \mathrm{~nm}, 519 \mathrm{~nm}, 563 \mathrm{~nm}$, $596 \mathrm{~nm}, 616 \mathrm{~nm}$, and $635 \mathrm{~nm}$.

Figure 7 shows the graphical representation of the results obtained from all seven LEDs. Unlike the absorbance spectroscopy technique where the instrument was first calibrated to produce absorbance representation of the films' colors, the results produced through LEDs based system were in raw voltage produced by the detector. The system basically was set up to measure the light transmitting through the film. Films with a darker color would have less light transmitting through them, and subsequently lower output voltage would be produced by the detector. The magnitude of light attenuation was depending on the wavelength of the LED. Similar to spectroscopy results, illumination light with a peak wavelength at $635 \mathrm{~nm}$ showed the best sensitivity in profiling the color changes of EBT3 films and subsequently the solar UV exposure on the films. By comparable observation of the spectroscopy technique, the highest sensitivity of the wavelength could be seen only for UV exposure lower than $10000 \mathrm{~mJ} / \mathrm{cm}^{2}$. Much lower responsivity, however, was recorded especially for exposure measurement above $15000 \mathrm{~mJ} / \mathrm{cm}^{2}$. Wavelengths of $616 \mathrm{~nm}$ and $596 \mathrm{~nm}$ exhibited a similar response to each other while wavelengths of $501 \mathrm{~nm}$ and $462 \mathrm{~nm}$ began to display higher linearity.

In this experiment, blue illumination light with a peak wavelength at $462 \mathrm{~nm}$ retained its relatively lower sensitivity among all other wavelengths for low UV exposure measurement. Regardless of that, 
for the entire measurement of solar UV exposure, the spectroscopy and LED-based system showed the ability of wavelength within the blue region in maintaining its sensitivity throughout the measurement. This can be observed through the linear relationship of the response that can be represented by a single responsivity (i.e., slope of the linear graph). While many previous studies that utilized red light in film analysis showed an early decline in sensitivity when measuring large UV exposure, in this experiment, the application of blue LED, on the other hand, may increase the ability of the optical system to profile films with much higher exposure. Other than blue light, based on the result in Fig. 5, red light with $650 \mathrm{~nm}$ wavelength could also be considered for high dose measurement as this wavelength did not show saturation (i.e., near optimum light absorption by the film) at a higher dose (above $10000 \mathrm{~mJ} / \mathrm{cm}^{2}$ ), where the linear response and sensitivity were in fact much higher than blue wavelength.

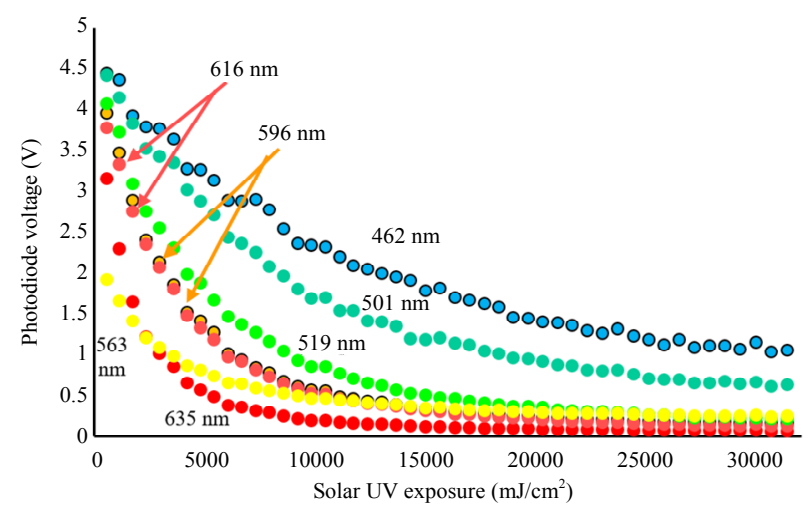

Fig. 7 Response curve obtained from the relationship between the solar ultraviolet exposures (in $\mathrm{mJ} / \mathrm{cm}^{2}$ ) and output voltage of photodiode through the individual illumination by seven light emitting diodes with distinctive peak emission wavelengths between $462 \mathrm{~nm}$ and $635 \mathrm{~nm}$.

Table 4 lists the functions used in generating regression fit across data and their respective $R^{2}$. For this experiment, only the reciprocal function was used to profile results generated by using all LEDs. The high accuracy of fitting was produced with $R^{2}$ above 0.99 , indicating the suitability and high accuracy of the functions used in representing the response curve.

Table 4 Summary of functions and coefficients of determination obtained from the relationship between the solar ultraviolet exposures (in $\mathrm{mJ} / \mathrm{cm}^{2}$ ) and output voltage of photodiode through individual illumination by seven light emitting diodes with distinctive peak emission wavelengths between $616 \mathrm{~nm}$ and $635 \mathrm{~nm}$.

\begin{tabular}{|c|c|c|c|}
\hline Function & Wavelength (nm) & Coefficients & $R^{2}$ \\
\hline \multirow{7}{*}{$\begin{array}{l}\text { Reciprocal } \\
y=\frac{a}{x-h}+k\end{array}$} & $\begin{array}{c}462 \\
\text { (Blue LED) }\end{array}$ & $\begin{array}{c}a=58503.604 \\
h=-11373.696 \\
k=-0.397\end{array}$ & 0.996 \\
\hline & $\begin{array}{c}501 \\
\text { (Cyan LED) }\end{array}$ & $\begin{array}{c}a=37768.399 \\
h=-6982.772 \\
k=-0.454\end{array}$ & 0.996 \\
\hline & $\begin{array}{c}519 \\
\text { (Green LED) }\end{array}$ & $\begin{array}{c}h=-3098.769 \\
k=-0.372\end{array}$ & 0.995 \\
\hline & $\begin{array}{c}563 \\
\text { (Yellow LED) }\end{array}$ & $\begin{array}{c}a=5005.791 \\
h=-2050.865 \\
k=+0.063\end{array}$ & 0.998 \\
\hline & $\begin{array}{c}596 \\
\text { (Orange LED) }\end{array}$ & $\begin{array}{c}a=10971.238 \\
h=-1889.459 \\
k=-0.267\end{array}$ & 0.991 \\
\hline & $\begin{array}{c}616 \\
\text { (Red - Orange } \\
\text { LED) }\end{array}$ & $\begin{array}{c}a=10844.246 \\
h=-1953.438 \\
k=-0.288\end{array}$ & 0.991 \\
\hline & $\begin{array}{c}635 \\
\text { (Red LED) }\end{array}$ & $\begin{array}{c}a=2886.578 \\
h=-315.083 \\
k=-0.058\end{array}$ & 0.996 \\
\hline
\end{tabular}

\section{Conclusions}

This research has shown that an LED-based optical system can be used to profile the color changes of EBT3 films. The results obtained are comparable to those from the visible spectrometer. Red wavelength, especially around $635 \mathrm{~nm}$, is commonly considered the most sensitive in analyzing EBT3 films. Since the relationship between the color changes of EBT3 films and UV exposure is according to asymptotic functions, the wavelength is only highly sensitive up to a certain level of exposure. Beyond that, the relationship curve will start to gradually move towards asymptote before saturating. While for blue wavelength, though considered to be the most ineffective wavelength, it can retain relatively constant sensitivity through the measurement of EBT3 films with large UV exposure. The results from this experiment are suitable to be 
implemented for ionizing radiation exposure such as high energy X-rays, except the color of the films needs to be recalibrated accordingly to the radiation dose.

\section{Acknowledgment}

This work was financially supported by the Ministry of Higher Education Malaysia Fundamental Research Grant Scheme (Grant No. 203 / PFIZIK / 6711491).

Open Access This article is distributed under the terms of the Creative Commons Attribution 4.0 International License (http://creativecommons.org/licenses/by/4.0/), which permits unrestricted use, distribution, and reproduction in any medium, provided you give appropriate credit to the original author(s) and the source, provide a link to the Creative Commons license, and indicate if changes were made.

\section{References}

[1] V. C. Borca, M. Pasquino, G. Russo, P. Grosso, D. Cante, P. Sciacero, et al., "Dosimetric characterization and use of GAFCHROMIC EBT3 ilm for IMRT dose verification," Journal of Applied Clinical Medical Physics, 2013, 14(2): 158-171.

[2] C. Y. P. Ng, S. L. Chun, and K. N. Yu, "Quality assurance of alpha-particle dosimetry using peeled-off gafchromic EBT3® film," Radiation Physics and Chemistry, 2016, 125: 176-179.

[3] K. Chelminski, B. Buczek, and W. Bulski, "EP-1366: comparison of gafchromic EBT, EBT2, and EBT3 radiochromic films when used with flat bed scanners,' Radiotherapy and Oncology, 2015, 115: S736.

[4] M. J. Butson, T. Cheung, P. K. N. Yu, and H. Alnawaf, "Dose and absorption spectra response of EBT2 gafchromic film to high energy X-rays," Australasian Physical and Engineering Sciences in Medicine, 2009, 32(4): 196-202.

[5] D. Welch, H. M. Spotnitz, and D. J. Brenner, "Measurement of UV emission from a diffusing optical fiber using radiochromic film," Photochemistry and Photobiology, 2017, 93(6): 15091512.

[6] T. A. D. Brown, K. R. Hogstrom, D. Alvarez, K. L. Matthews, K. Ham, and J. P. Dugas, "Dose-response curve of EBT, EBT2, and EBT3 radiochromic films to synchrotron-produced monochromatic X-ray beams," Medical Physics, 2012, 39(12): 1-18.

[7] M. Callens, W. Crijns, V. Simons, I. De Wolf, T. Depuydt, F. Maes, et al., "A spectroscopic study of the chromatic properties of EBT3 gafChromic films," Medical Physics, 2016, 43(3): 1156-1166.

[8] M. A. Tajuddin and A. F. Omar, "Measuring solar ultraviolet exposure dose on EBT3 film through the application of visible absorbance spectroscopy," in 9th International Conference on Robotic, Vision, Signal Processing and Power Applications, Singapore, 2017, pp. 639-646.

[9] S. L. Chun and P. K. N. Yu, "Note: calibration of EBT3 radiochromic film for measuring solar ultraviolet radiation," Review of Scientific Instruments, 2014, 85(10): 106103.

[10] D. Welch, G. Randers-Pehrson, H. M. Spotnitz, and D. J. Brenner, "Unlaminated gafchromic EBT3 film for ultraviolet radiation monitoring," Radiation Protection Dosimetry, 2017, 176(4): 341-346.

[11] T. Katsuda, R. Gotanda, T. Gotanda, T. Akagawa, N. Tanki, T. Kuwano, et al., "Intensities of incident and transmitted ultraviolet - a rays through gafchromic films," Journal of Medical Physics, 2017, 42(2): 86-89.

[12] N. A. M. Yusof, U. S. Osman, and A. F. Omar, "Measuring UV LEDs radiation dose using EBT3 film," in 2016 International Conference on Advances in Electrical, Electronic and Systems Engineering (ICAEES), IEEE, Putrajaya, Malaysia, 2016: 5-10.

[13] E. T. Butson, T. Cheung, P. K. N. Yu, and M. J. Butson, "Measuring solar UV radiation with EBT radiochromic film," Physics in Medicine \& Biology, 2010, 55(20): N487-N493.

[14] K. N. Yu, S. L. Chun, and P. M. Chan, "Long-term passive monitoring of solar UV radiation using radiochromic films," in 3rd Southern African Solar Energy Conference, South Africa, 2015, pp. 463465.

[15] P. Y. Tsang, P. M. Chan, and K. N. Yu, "Results in physics measuring diffuse ultraviolet exposures using gafchromic EBT3 films," Results in Physics, 2017, 7: 1492-1493.

[16] K. N. Yu, S. L. Chun, and P. M. Chan, "Results in physics responses of gafchromic EBT3 films with polypropylene barriers to UV radiation," Results in Physics, 2017, 7: 1976-1977.

[17] A. Aydarous, E. A. Al-omary, and M. El Ghazaly, "Characterization of gafchromic EBT3 films for ultraviolet radiation dosimetry," Radiation Effects and Defects in Solids, 2014, 169(3): 249-255.

[18] N. Farah, Z. Francis, and M. Abboud, "Analysis of the EBT3 gafchromic film irradiated with $6 \mathrm{MV}$ photons and $6 \mathrm{MeV}$ electrons using reflective mode scanners," Physics Medical, 2014, 30(6): 708-712.

[19] L. Richley, A. C. John, H. Coomber, and S. Fletcher, "Evaluation and optimization of the new EBT2 radiochromic film dosimetry system for patient dose verification in radiotherapy," Physics in Medicine \& Biology, 2010, 55(9): 2601-2617.

[20] M. J. Williams and P. E. Metcalfe, "Radiochromic 
film dosimetry and its applications in radiotherapy," AIP Conference Proceedings, 2011, 1345(1): 75-99.

[21] M. B. Callens, W. Crijns, T. Depuydt, K. Haustermans, F. Maes, E. D'Agostino, et al., "Modeling the dose dependence of the vis-absorption spectrum of EBT3 GafChromic films," Medical Physics, 2017, 44(6): 2532-2543.

[22] S. Park, S. K. Kang, K. H. Cheong, T. Hwang, H. Kim, T. Han, et al., "Variations in dose distribution and optical properties of gafchromic ${ }^{\mathrm{TM}}$ EBT2 film according to scanning mode," Medical Physics, 2012, 39(5): 2524-2535.

[23] E. Y. León Marroquin, J. A. Herrera González, M. A. Camacho López, J. E. Villarreal Barajas, and O. A. García-Garduño, "Evaluation of the uncertainty in an EBT3 film dosimetry system utilizing net optical density," Journal of Applied Clinical Medical Physics, 2016, 17(5): 466-481.

[24] S. Aldelaijan and S. Devic, "Comparison of dose response functions for EBT3 model GafChromic ${ }^{\mathrm{TM}}$ film dosimetry system," Physics Medical, 2018, 49:
$112-118$.

[25] E. Spelleken, S. B. Crowe, B. Sutherland, C. Challens, and T. Kairn, "Accuracy and efficiency of published film dosimetry techniques using a flat-bed scanner and EBT3 film," Australasian Physical \& Engineering Sciences in Medicine, 2018, 41(1): 117 128.

[26] E. Butson, H. Alnawaf, P. K. N. Yu, and M. Butson, "Scanner uniformity improvements for radiochromic film analysis with matt reflectance backing," Australasian Physical \& Engineering Sciences in Medicine, 2011, 34(3): 401-407.

[27] A. F. Omar and M. Z. Matjafri, "Specialized optical fiber sensor for nondestructive intrinsic quality measurement of averrhoa carambola," Photonic Sensors, 2013, 3(3): 272-282.

[28] TAOS Inc. Texas Advanced Optoelectronic Solutions, "TSL257 High-sensitivity light-to-voltage converter," Technical Datasheet. TAOS023E September 2007. 